\title{
MANUS Canada Annual Scientific Meeting
}

June 15, 2006

Quebec City, Quebec

\section{1}

\section{A COMPARISON OF OBJECTIVE ABDUCTOR POLLICIS BREVIS STRENGTH TESTING AND NERVE CONDUCTION STUDIES IN THE DIAGNOSIS OF CARPAL TUNNEL SYNDROME \\ DS McDonald, MSG Bell \\ Ottawa, Ontario}

Currently, nerve conduction studies (NCS) are the gold standard for objective quantification of median nerve damage in carpal tunnel syndrome (CTS). However, NCS are not perfect. Many patients presenting with a clinical picture of CTS do not have abnormal NCS findings but may benefit from carpal tunnel release (CTR). To this date, no study has addressed the relative utility of objective, quantitative measurement of abductor pollicis brevis (APB) strength in the diagnosis of CTS. Specifically, no one has compared the results of objective APB strength measurement (OAPBM) with NCS results. In this study, we have compared OAPBM with NCS measures in 50 patients with CTS. We show that the correlation between APB strength and "severity" of CTS as determined by NCS $(\mathrm{r}=-0.19)$ is statistically insignificant $(\mathrm{p}=0.22)$. However, we also show that 8 patients who were "normal" according to NCS and "impaired" according to OAPBM showed improvement in APB strength following CTR (suggesting that they were missed by NCS). None of the patients considered to have CTS by NCS were missed by OAPBM. Additionally, we have found significant reductions in $\mathrm{APB}$ strength in patients considered to have even "mild" CTS by NCS. Clinical use of OAPBM in the diagnosis of CTS is appealing for several reasons. For example it is much less expensive, more accessible, and more rapid than NCS.

\section{2}

PATIENT SATISFACTION WITH THE CHOICE OF ANESTHETIC TECHNIQUES AND SETTINGS FOR OPEN CARPAL TUNNEL RELEASE

V Panchapakesan, L Kasrai, A EIMaraghy

St Joseph's Hospital, Toronto, Ontario

PURPOSE: Open carpal tunnel release (OCTR) is one of the most common outpatient surgical procedures performed in Ontario. Wide variability exists in the choice of anesthetic technique [general anesthesia (GA), intravenous regional anesthesia (IVRA) or local anesthesia (LA)] and setting [main operating room (OR) or minor surgery (MS)] for this procedure. The impact of these choices on the patient's experience during and after surgery has not been previously explored. The purpose of this study was to investigate differences in patient satisfaction between various anesthetic techniques and surgical setting for OCTR.

METHODS: A retrospective review of patients undergoing carpal tunnel release at St Joseph's Health Centre in Toronto, Ontario was conducted. A total of 125 procedures were reviewed, with comparable numbers from each of four major categories (A) GA in OR, B) IVRA in OR, C) LA in OR, D) LA in MS]. Patient demographics were similar amongst all groups. We used the Iowa Satisfaction with Anesthesia Scale (ISAS), a validated patient questionnaire, as our outcome measure. These data were subjected to statistical analysis.

RESULTS: Statistical analysis demonstrated differences in ISAS scores between all groups. Patient satisfaction scores are provided. No statistical differences in patient demographics were found.

CONCLUSIONS: There are potential differences in patient satisfaction with regard to the type of anesthetic technique and surgical setting chosen for OCTR. Although the ideal choice of anesthesia and setting for OCTR will depend on a number of factors - including surgeon training and preference, and resource availability in each hospital - surgeons should consider these patient satisfaction differences when planning for OCTR.

3

\section{EXPERIENCE WITH WRIST FUSION IN 47 PATIENTS WITH} BRACHIAL PLEXUS INJURY

IK Terzis, I Kostas, H Molligan IV

Eastern Virginia Medical School, Norfolk, Virginia

Objectives: Devastating brachial plexus injury commonly results in a frail upper extremity. Hand reanimation efforts benefit from stabilization of the wrist joint due to paucity of adequate motors. The purpose of this study is to present the indications for wrist fusion, determine the union rate, and analyze the impact on overall function.

METHODS: From 1981 to 2001, 47 patients with brachial plexus palsy (BPP) underwent wrist fusion at the International Institute of Reconstructive Microsurgery. Thirty-nine (39) were males and eight (8) were females, with ages ranging from 17 to 54 . The AO/ASIF Titanium wrist fusion plate was used with bone autograft or allograft. A volar Plaster of Paris slab was applied for 4 weeks, and the wrist was further protected with a custom-made wrist splint for an additional 8 weeks. Anteroposterior and lateral wrist radiographs were taken at 3, 6, 9, 12, and 18 months after surgery in order to evaluate the union rate. The minimum follow-up was 24 months, with a mean follow-up of 35 months.

RESULTS: Wrist fusion occurred at a mean of 12 weeks in all 47 wrists. The average position of wrist fusion was 22 degrees of extension. There was no evidence of loosening of the screws or the plate. In all the patients the hand function was improved.

CONCLUSIONS: The wrist fusion procedure in BPP restores a stable wrist joint, improves function and appearance of the upper extremity, giving also a prop for a bi-manual grip. Wrist arthrodesis is necessary to liberate any functioning wrist motors so they can be transferred to the fingers allowing improved grip strength in the reanimated hand.

\section{4}

\section{BASILAR JOINT OSTEOTOMY IN THE TREATMENT OF OSTEOARTHRITIS: LONG TERM OUTCOMES OF A MINIMALLY INVASIVE PROCEDURE}

WL Parker, RL Linscheid, PC Amadio

Department of Orthopedics, Mayo Clinic, Rochester, Minnesota PURPOSE: Basal first metacarpal osteotomy, a minimally invasive treatment, is an often overlooked option for treating trapeziometacarpal osteoarthritis. Our purpose was to review the Mayo Clinic experience with Wilson osteotomy for the management of basilar joint arthritis to determine its effectiveness and durability.

METHODS: We retrospectively reviewed 8 patients, treated by a single surgeon by basal metacarpal osteotomy. Data were collected from medical 
records, and Eaton stage was determined from pre-operative and final radiographs.

RESULTS: All patients were female, average age of 48.8 years. Mean follow up was 9 years $(6.2-13.4)$. All were right hand dominant, with the left hand involved in 6/8 cases. All osteotomies united primarily. Average appositional pinch, oppositional pinch and grip measured as $4.7 \mathrm{~kg}, 3.1 \mathrm{~kg}$, and $19.4 \mathrm{~kg}$ respectively $(129.3 \%, 103.4 \%, 107.9 \%$ of contralateral). Eaton stage was preserved in $5 / 8$ patients with excellent functional outcomes in $6 / 8$ patients at 8.7 years. Positive outcomes were not correlated to preoperative Eaton stage, change in stage, or post-operative strength achieved. 2 patients underwent subsequent procedures; 1 at 6.6 years and the other at 13.4 years, both with a prolonged pain free interval prior to presentation for re-operation.

CONCLUSIONS: Our data demonstrate the effectiveness and durability of the basal metacarpal osteotomy and supports this treatment strategy in early and moderate Eaton stages, conferring minimal morbidity without interference with future salvage procedures.

\section{5}

\section{SCAPHO-LUNATE DISSOCIATION, EXPERIENCE WITH RECONSTRUCTION WITH A MODIFIED BRUNELLI TECHNIQUE}

D Cloutier, C-E Ferron

Laval University, Quebec City, Quebec

Scapho-lunate dissociation remains a difficult problem to treat adequately. We have treated 4 patients with a modification of Brunelli's original technique. Half of the flexor carpi radialis tendon is transposed through the scaphoid and supplemented with fixation with a loop around the radiotriquetral ligament. The pathophysiology of these injuries will be reviewed and the results of our experience will be presented.

\section{6}

ORTHOSPHERE INTERPOSITIONAL ARTHROPLASTY FOR TRAPEZIOMETACARPAL OSTEOARTHRITIS.

T Best, G Elder

PURPOSE: To report early clinical results of the orthosphere interpositional arthroplasty (Wright Technology Inc, Arlington, TN) and the pathological findings in failed cases.

METHODS: A retrospective review of 8 patients who underwent implantation of an orthosphere interposition between 2002-2004 was conducted. The indication for surgery was first carpometacarpal osteoarthritis non responsive to conservative measures. In cases where revision occurred, excised trapezial specimens were sent to a bone pathologist to determine a possible cause of failure of the implant.

RESULTS: Six of 8 patients showed radiological evidence of significant subsidence (up to $8 \mathrm{~mm}$ ). Five patients underwent revision surgery (FCR interpositional arthroplasty) for recurrent pain and disability. Another patient remained symptom-free with a good result at 1-year follow up despite subsidence of $7 \mathrm{~mm}$, and the 2 remaining patients had good clinical results at a mean 18 months follow up although one showed a $2 \mathrm{~mm}$ radiolucent line around the entire implant but no subsidence. Pathology review of the 3 revised failures showed only compressive effects on the trapezial bony trabeculae with associated inflammatory reaction of synovial tissues. No third body wear particles were documented nor was there evidence of osteolysis.

CONCLUSIONS: Our clinical results were a $75 \%$ failure rate within 30 months leading to revision surgery. Mode of failure based on review of pathological specimens appeared to be mechanical rather than material wear. These results independently confirm the disappointing outcomes reported by Athwal et al for the orthosphere interpositional arthroplasty. As such, routine use of this device for basal thumb osteorarthritis cannot currently be recommended.

\section{7}

\section{TWO HUNDRED FLEXOR TENDON REPAIRS OF THE HAND TREATED AT SAINT VINCENT'S UNIVERSITY HOSPITAL, DUBLIN AND REHABILITATED USING EARLY ACTIVE MOBILIZATION}

\section{SM Carroll, H Al Shah}

PURPOSE: This study aims to evaluate the outcome of flexor tendon repairs of the hand rehabilitated using early active mobilization (EAM) over the last five years. EAM has been in use at Saint Vincent's University Hospital for the last ten years.

METHOD: All patients who had flexor tendon repairs in the last five years were identified retrospectively. Two hundred flexor tendon repairs of the hand were performed. Of these 48 tendons repaired in 34 patients were performed in 2005 and were initially analyzed. These results are presented in this abstract. A further 152 results will be analyzed and presented at the meeting. All patients were managed post-operatively with a dorsal blocking splint and an EAM regime which incorporated immediate active flexion and extension for tendon rehabilitation. Follow-up assessments included range of motion, grip strength, complications, time of return to normal activities and functional outcome. Results are based on the American Society for Surgery of the Hand criterion.

RESULTS: Functional results for the tendon repairs were as follows: excellent:22, good:16, fair:4, poor:2. Examination of flexion deficit: excellent:31, good:6, fair:1, poor:4 and measurements of extension lag $1-15^{\circ}: 18$, $16-30^{\circ}, 31-50^{\circ}: 7$ and $>50^{\circ}: 1$

CONCLUSIONS: EAM of these patients has produced an excellent functional outcome with the majority of patients returning to their normal daily activities within three months of tendon repair.

\section{8}

\section{PERCUTANEOUS TRIGGER FINGER RELEASE: A NOVEL INSTRUMENT AND TECHNIQUE}

D Rowe, V Vijitpornkul, PP Narini, HS Matloub

Department of Plastic Surgery, Medical College of Wisconsin, Milwaukee, Wisconsin

INTRODUCTION: Triggering of the digit is one of the most common causes of pain and disability seen by the hand surgeon. Despite many techniques described over the past 40 years, percutaneous release of the triggering digit has not gained widespread acceptance in the United States. We describe a novel instrument and technique for the percutaneous release of trigger digits.

METHODS: The novel probe used in this analysis is a modified dental probe with two distinct blades; a blade located parallel to the instrument and a blade located perpendicular to the instrument. A retrospective longterm follow up study was instituted on 1,000 consecutive patients undergoing percutaneous release. Eight hundred and eighty-nine patients (out of 1,000 ) were contacted via phone interview or personal visit between four months and one year postoperatively. Patients were asked a series of questions regarding use of the digit, persistent pain, evidence of neurovascular injury (discoloration, numbness, or tingling) of the digit, and bowstringing. A short-term prospective evaluation of 200 consecutive patients was also performed. Patients were evaluated at seven and 14 days postprocedure. Evaluation consisted of examination for residual triggering, evidence of vascular injury, neurological injury via Semmes-Weinstein monofilament test, evidence of bowstringing of the flexor tendon, and pain.

RESULTS: All digits treated had complete resolution of triggering with no instances of recurrence or need for open release. In the long-term retrospective analysis, no recurrent triggering, persistent pain, bowstringing, or neurovascular injury were found. In the short-term prospective analysis of 200 patients, mild pain with movement was experienced by $9 \%$ of patients and mild bruising by $14 \%$ of patients at the first postoperative visit, but were resolved by the time of the two-week exam. No instances of neurovascular injury or tendon bowstringing were reported.

CONCLUSION: We report a novel technique and instrument for the safe and effective release of the A1 pulley in the instance of the triggering digit. No evidence of neurovascular or tendon injury and no recurrence was seen in this large series. 
9

ULNO-TRIQUETRAL LIGAMENT SPLIT TEARS: UNRAVELLING ULNAR SIDED WRIST PAIN.

WL Parker, RA Berger

Department of Orthopedics, Mayo Clinic, Rochester, Minnesota

PURPOSE: Ulnar sided wrist pain is analogous to lower back pain; symptoms are vague, chronic, imaging often noncontributory, and treatment ill defined. We have defined a novel TFCC pathology - the ulno-triquetral ligament split tear. This is a longitudinal rent created within the substance of the ligament causing it to splay open. Our objectives were to identify: patient demographics, clinical presentation, mechanism of injury, imaging, and describe our management with early outcomes in a large patient series. METHODS: 36 patients were identified intraoperatively as having a U-T ligament split tear. All patients underwent an "outside-in" repair and their medical records were retrospectively reviewed.

RESULTS: Average age was 30 years, $47.2 \%$ were male, and $36.1 \%$ were competitive athletes. Right sided U-T tears comprised $69.4 \%$. When traumatic, the mechanism involved hyperextension/supination in $96.3 \%$. Average duration of symptoms was 14.9 months. 100\% reported ulnar sided wrist pain with pro/supination aggravating in $80.6 \%$. A positive "fovea sign" was found in $93.8 \%$. $70 \%$ had a signal change/fluid at the U-T ligament or foveal insertion of the TFCC on magnetic resonance imaging. Patient satisfaction and pain relief without functional limitations were achieved in $89.7 \%$. Grip strength and range of motion were near normal at 2.6 months. 2 patients went on to DRUJ instability requiring reconstruction. CONCLUSIONS: We have identified a novel TFCC injury, provided insight into its etiology and presentation, and demonstrated convincing outcomes of our surgical treatment. Thus, we furthered the understanding of TFCC pathology and aided in unravelling the complexities of ulnar sided wrist pain.

\section{0}

NON-CONSTRAINED PYROLYTIC CARBON IMPLANTS: EARLY OUTCOMES IN OSTEOARTHRITIC MP JOINTS

WL Parker, SL Moran, KB Hormel, M Rizzo, RD Beckenbaugh Department of Orthopedics, Mayo Clinic, Rochester, Minnesota PURPOSE: Osteoarthritis of the MP joint is relatively uncommon but when present can limit its function and cause significant pain. Our objective was to review the early outcomes of patients with primary and posttraumatic osteoarthritis treated at the Mayo Clinic with a new generation, non-constrained, pyrolytic carbon implant.

METHODS: 20 MP joint arthroplasties (18 patients) for OA over 3 years were reviewed. Patient questionnaires were completed pre-operatively, and at 6 weeks, 12 weeks, 6 months, and 1 year following surgery. Pain, function, and appearance were rated on visual analogue scales; satisfaction, ROM, and strength were documented at all time points.

RESULTS: The average patient age was 57 years. There were 9 index and 11 long fingers. Pre-operative radiographs showed $43 \%$ with subluxation and no dislocations. Average follow-up was 14 months (3-39 months). Oppositional pinch increased $125.9 \%(\mathrm{p}=0.02)$ and grip strength improved nearly $40 \%(\mathrm{p}=0.04)$. The ROM improved from $-21^{\circ}$ extension $/ 65^{\circ}$ flexion to $-15^{\circ}$ extension $/ 73^{\circ}$ flexion $(\mathrm{p}=0.17)$. Pain decreased from 73 to $8.5 / 100$ $(p=0.0004)$. Functionality increased from 20.1 to $86.6 / 100(p=0.0002)$. Cosmesis approval rose from 62.7 to $93.6 / 100$ ( $\mathrm{p}=0.01$ ). $75 \%$ of patients returned to previous employment. One extensor tendon rupture occurred and one case of chronic pain required implant removal. A normal radiolucent seam was seen in $100 \%$ of patients at one year follow-up.

CONCLUSIONS: Initial short-term results show excellent motion, pain relief and restoration of pinch and grip strength. These results are encouraging and suggest that pyrolytic carbon arthroplasty is an excellent option for joint salvage in patients suffering from primary and post-traumatic MP joint osteoarthritis.
11

BONE DENSITY COMPARISON OF SELECTED CARPAL AND TARSAL BONES: VALIDATION FOR THEIR USE IN COMPRESSION FRACTURE FIXATION STUDIES OF SCAPHOID SCREWS

IP Brutus, JS Rajkumar, E Rust, BJ Harley, AK Palmer, FW Werner

INTRODUCTION: To determine if trabecular, total and cortical bone densities of the capitate, navicular, cuboid, and first cuneiform were equivalent to those of the scaphoid, such that these bones could be used in place of the scaphoid in evaluating new headless scaphoid compression screws. METHODS: Fifty scaphoids, capitates, naviculars, cuboids, and first cuneiforms were harvested from fresh frozen cadavers. The trabecular, total and cortical bone densities were measured using pQCT technology and statistically compared.

RESULTS: A paired t comparison between paired scaphoids and capitates showed no difference between the trabecular bone densities. However, their total bone and cortical densities were found to be different. An independent measures ANOVA comparison of the five bones, showed no significant difference in mean trabecular density between the capitates, naviculars and first cuneiforms when compared to the scaphoids. However, the mean total and cortical densities of the first cuneiforms were less than those of the scaphoids and the mean trabecular, total, and cortical bone densities of the cuboids were all less than those of the scaphoids.

DISCUSSION: Compression fracture fixation studies of headless compression screws could be conducted using the capitate, navicular, and first cuneiform as models of the scaphoid when the supply of scaphoids is limited.

12

GERDY'S TUBERCLE AS A SOURCE OF CANCELLOUS BONE GRAFT FOR SURGERY OF THE UPPER EXTREMITY: DESCRIPTION OF TECHNIQUE AND EVALUATION OF ASSOCIATED MORBIDITY

IP Brutus, JB Loftus

Cancellous bone grafts can be harvested from Gerdy's tubercle on the proximal metaphysis of the tibia for surgical procedures on the upper extremity. However, this donor site is rarely used by hand surgeons, possibly by lack of awareness.

TECHNIQUE: Gerdy's tubercle is exposed through a short incision placed on the lateral aspect of the knee after identification of palpable landmarks. Dissection and muscle stripping are minimal. Harvest is performed after corticotomy with osteotomes and small curettes. Closure is performed in two layers. A bulky dressing is applied and full weight bearing allowed. Nineteen tibias were harvested for procedures requiring cancellous bone grafting of the upper extremity (partial and total wrist fusion, distal radius fixation or osteotomy). The volume and quality of cancellous bone grafts were adequate for all procedures. Unlimited mobilization and weight bearing was allowed in the immediate postoperative period. Complications (follow up of 41.6 months) included two sterile dehiscences but no hematoma, infection or fracture of the tibial plateau. Four patients reported some local discomfort at 6 weeks but with normal gait. Late follow up demonstrated normal range of motion and gait in all patients. Gerdy's tubercle is a reliable low morbidity donor site of cancellous bone grafts and is suitable for use in distal upper extremity recontructive procedures. It can be considered an alternative when the ipsilateral distal radius cannot be used as a donor site as in procedures on the distal radius itself.

\section{3}

CHALLENGES IN TREATING ENCHONDROMAS OF THE BONES OF THE HAND

NH McKee

University of Toronto, Toronto, Ontario

PURPOSE: This presentation will outline the distinguishing aspects of history, clinical findings, imaging and pathology that contribute to the decisions made about care of enchondromas and Stage 1 chondrosarcomas of the hand. Radiological follow up of enchondromas treated without any bone grafting will illustrate the adequacy of the treatment. 
METHODS: The presenter's personal experience will be combined with an updated review of the related literature. "Do no harm" was the principle behind care. Operative treatment was not initiated until full function was restored. Surgery consisted of thorough curettage of the lesions through a small cortical window. For most of the presenter's career, no bone grafts or other substitutes have been used. Outcome is judged by clinical function and $\mathrm{x}$-rays.

RESULTS: Most lesions are enchondromas. Grade 1 chondrosarcoma lesions are rare. Presentation of enchondroma was often with a pathological fracture. Healing of the fractures and restoration of full function preceded elective surgery. Post operative follow up $\mathrm{x}$-rays document the disappearance of cystic lesions in the bone illustrating the capacity of the bone to restore itself. A few patients with enchondromas have chosen not to have surgery and have pursued normal activities for years.

CONCLUSIONS: Treatment focussed at each step to maintain and restore function has yielded normally functioning fingers. Follow up $\mathrm{x}$-rays after thorough curetting of enchondromas without bone grafting show loss of cystic appearance. Grade 1 chondrosarcomas can be treated initially with meticulous curettage and finger preservation but must have follow up.

\section{4}

\section{A COMPARISON OF DISTAL ZONE II FLEXOR TENDON SUPERFICIALIS REPAIR TECHNIQUES}

A Yazdani, R Stacpoole, L Ferreira, S Chinchalkar, D Ross Division of Plastic Surgery, Hand \& Upper Limb Centre, University of Western Ontario, London, Ontario

PURPOSE: The purpose of this study was to assess the mechanical strength of various tendon suture techniques in the repair of flexor digitorum superficialis (FDS) tendons.
METHODS: Four groups (6-8/group) of human FDS tendons were harvested. Each was transected and then repaired with 1 of 4 previously described techniques: horizontal mattress, Tajima, Bunnell, and Krakow. Tendons were mounted using custom clamps on an Instron materials testing machine and ramp loaded at $4 \mathrm{~mm} /$ minute. Force required to produce gapping and ultimately rupture was recorded. Results were compared using ANOVA.

RESULTS: The Bunnell repair was strongest $(\mathrm{p}<0.05)$ in resisting both gapping and rupture. The Tajima and Krakow suture techniques were significantly stronger than the horizontal mattress suture but not different from one another. The horizontal mattress suture was weakest of all techniques. The Krakow technique failed at the suture itself as opposed to pulling through the tendon substance.

CONCLUSIONS: The Bunnell suture technique is the mechanically strongest method of the 4 techniques tested. This study does not address the questions of gliding nor the potential strangulation of the micro-circulation that has been raised as a criticism of the Bunnell method.

\section{BOOK REVIEWS}

Continued from page 89

it is often difficult to emerge with a specific treatment plan without the benefit of a comprehensive overview, perhaps a consequence of too many opinions, too many choices.

I would find this a difficult text to read cover to cover. However, it would prove a useful resource for practicing surgeons and residents with a solid foundation of oculofacial plastic surgery.

Dr JB Baum MD FRCSC Division of Plastic Surgery, Department of Surgery William Osler Health Centre, Ontario

Philip N Blondeel, Steven F Morris, Geoffrey G Hallock, Peter C Neligan, eds. Perforator Flaps: Anatomy, Technique, and Clinical Applications, Volume 1, 2006. Quality Medical Publishing Inc, ISBN 1-57-626178-6, $1000 \mathrm{pp}$, US\$470.00

This textbook is sure to become a standard reference text for students of plastic surgery, and for all plastic surgeons interested in microsurgery.

Volume 1 of 2 was reviewed. It appears to be a good looking text which is logically laid out, with superior diagrams, illustrations and photographs. The book is the work of many notable microsurgeons from around the world, authoring different chapters. The editorial group has arranged the chapters in a logical sequence, which adds to the effective organization of the text.
Volume 1 begins with a proposed standardization for nomenclature of perforator flaps. Following this, an introduction by G Ian Taylor briefly recounts the history of flap reconstruction that has brought us to the microsurgical evolution of perforator flaps.

The first volume of this text is divided into two main parts. The first section is a review of fundamentals, such as anatomy, classification, physiology, preoperative planning and avoiding complications. The second section of volume 1 reviews regional flaps and surgical techniques of the head and neck, upper extremity and trunk.

Volume 2, although not reviewed, looks at regional flaps and surgical techniques of the lower extremities, clinical applications and future directions to complete the textbook.

The text is very readable, well organized and logical in its approach to perforator flaps and their usage. The anatomy and applicability of these flaps is well demonstrated. The text also includes an educational DVD, which illustrates the anatomy and elevation of the deep inferior epigastric perforator (DIEP) flap, thoracodorsal artery perforator (TAP) flap and the ALT (lateral circumflex remoral artery) perforator flap. This DVD is easy to follow and of excellent quality.

I would highly recommend this textbook to all plastic surgery training programs, residents and all those with an interest in microsurgery.

Ken Murray MD FRCSC Section of Plastic Surgery University of Manitoba, Winnipeg, Manitoba 